\title{
ORIGINAL
}

\section{CALIDAD DE VIDA RELACIONADA CON LA SALUD COMO VARIABLE EXPLICATIVA DEL CONSUMO DE CONSULTAS EN ATENCIÓN PRIMARIA: UN ANÁLISIS POR SEXO (*)}

\section{Emilio Pariente Rodrigo (1), Ana Belén García-Garrido (2), Francisca Gómez Molleda (3), Encarnación Gálvez Castillo (4), Josefina González Expósito (5), Isabel López León (6), Patricia Rojo Cárdenas (7) y Giusi Alessia Sgaramella (8).}

(1) Centro de Salud Camargo-Interior. Muriedas. Cantabria.

(2) Centro de Salud Suances. Suances. Cantabria.

(3) Consultorio de Limpias. CS Bajo Asón. Ampuero. Cantabria.

(4) Centro de Salud Vargas. Santander.

(5) Centro de Atención a la Dependencia de Cueto. Instituto Cántabro de Servicios Sociales. Santander.

(6) Centro de Salud. Zabalgana. Osakidetza-Vitoria.

(7) Centro de Salud. Playa Blanca. Yaiza. Lanzarote.

(8) Servicio de Hospitalización Domiciliaria. Hospital Universitario Marqués de Valdecilla. Santander.

Los autores declaran no tener ningún conflicto de intereses

\section{RESUMEN}

Fundamentos: Es conocida la asociación inversa que existe entre la calidad de vida relacionada con la salud (CVRS) y el consumo de consultas. Sin embargo, el sexo es considerado habitualmente como variable de confusión, y son escasos los estudios que han analizado explícitamente las diferencias de sexo en esta cuestión. El objetivo fue conocer la influencia de la CVRS sobre el uso de consultas de Atención Primaria en las personas $>65$ años, en un análisis diferenciado por sexo.

Métodos: La selección de participantes se realizó a lo largo de 2013 , 191 mujeres y 155 varones de $\geq 65$ años fueron estudiados mediante entrevista y análisis de historias clínicas. Se utilizó el Cuestionario EuroQol-5D y fueron analizadas variables demográficas, clínicas y de apoyo social. Se elaboraron 2 modelos de regresión múltiple, uno dirigido a las mujeres y otro a los varones.

Resultados: La CVRS se correlacionó de forma negativa con el consumo de consultas (Rho-Spearman $=-0,22 ; \mathrm{p}=0,0001$ ), con una OR cruda de 1,85 (IC95\%: 1,2-2,9). La asociación se mantuvo significativa al ajustar tanto por variables demográficas $[\mathrm{OR}=1,99$ (IC95\%: 1,2-3,2)], como por variables clínicas $[\mathrm{OR}=1,79(\mathrm{IC} 95 \%: 1,1-2,9)]$ o de apoyo social $[\mathrm{OR}=1,83$ (IC95\%: 1,1-2,9)]. En el análisis de regresión, los valores del coeficiente estandarizado ( $\beta$ ) asociado a la CVRS fueron 0,22 (IC95\%: - 36,7- -6,9) en las mujeres, y $0,03(-15,6-23,1)$ en los varones.

Conclusiones: En las mujeres de $\geq 65$ años la CVRS es la variable con mayor capacidad explicativa de consumo de consultas, tras ajustar por variables demográficas, clínicas y de apoyo social. Por el contrario, en los varones su influencia es prácticamente nula.

Palabras clave: Calidad de vida. Anciano. Visita a consultorio médico. Género y salud. Atención Primaria de Salud.

Correspondencia

Emilio Pariente Rodrigo

Centro de Salud "Camargo-Interior"

Avda Bilbao, s/n

39600-Muriedas. Camargo.

Cantabria

emilio.pariente@scsalud.es

\section{ABSTRACT}

Health-Related Quality of Life as an Explanatory Variable of Primary Care Consultations: Sex Differences

Background: It is well known the inverse relationship between healthrelated quality of life (HRQoL) and the use of consultations. However, most studies deal sex as a confounding variable rather than to explicitly investigate sex differences. The study aims to know the influence of HRQoL of the elderly on the use of Primary Care consultations in a sex analysis.

Methods: Throughout 2013,191 women and 155 men aged 65 years or older were enrolled in the study and assessed with interviews and analysis of medical records. We used the EuroQol-5D to assess the HRQoL and several demographic, clinical and social support variables were also analyzed. Two multiple linear regression models were developed.

Results: HRQoL showed a negative correlation with the use of consultations (Spearman's rho $=-0,22 ; \mathrm{p}=0,0001$ ) and a crude $\mathrm{OR}$ value of 1,85 (95\% CI:1,2-2,9). The association remained significant after adjusting for demographic $[\mathrm{OR}=1,99(95 \% \mathrm{CI}: 1,2-3,2)]$, clinical $[\mathrm{OR}=1,79$ $(95 \% \mathrm{CI}: 1,1-2,9)]$ or social support covariates $[\mathrm{OR}=1,83(95 \% \mathrm{CI}$ : $1,1-2,9)]$. In regression analysis, the values of standardized coefficient $(\beta)$ related to HRQoL were $0,22(95 \% \mathrm{CI}:-36,7--6,9)$ in females and 0,03 $(95 \%$ CI:-15,6-23,1) in males.

Conclusions: In women $\geq 65$ years, HRQoL shows the greatest explanatory power of use of consultations, after adjusting for demographic, clinical and social support covariates. By contrast, its influence on men is negligible.

Keywords: Quality of life. Elderly. Office visits. Sex differences. Primary health care. 


\section{INTRODUCCIÓN}

La evaluación de la calidad de vida relacionada con la salud (CVRS) representa el impacto que una enfermedad y su tratamiento tienen sobre la percepción del paciente de su bienestar ${ }^{1}$. Patrick y Erickson definen la CVRS como la medida en que se modifica el valor asignado a la duración de la vida en función de la percepción de limitaciones físicas, psicológicas, sociales y de disminución de oportunidades a causa de la enfermedad, sus secuelas, el tratamiento y/o las políticas de salud ${ }^{2}$.

La CVRS aporta una información relevante sobre el estado de salud en las enfermedades crónicas, ha demostrado ser útil en la evaluación de la calidad asistencial y puede ser de ayuda en la toma de decisiones clínicas ${ }^{1,3}$. Además, una mala valoración de la CVRS es predictora de mortalidad y de un mayor consumo de servicios sanitarios ${ }^{4,5}$. En los estudios sobre la CVRS es frecuente observar diferencias notables entre ambos sexos. Así, las mujeres suelen referir una peor valoración de su CVRS, observación que se repite sistemáticamente en diferentes países y entornos socioculturales ${ }^{6,7}$.

Por otro lado, la población anciana consume una gran cantidad de recursos sanitarios. Según datos de nuestro país, el 54\% de las personas mayores de 65 años ha acudido a una consulta médica en las últimas 4 semanas ${ }^{8}$. La extensión y el volumen de estas consultas, unido al hecho de que no se conocen en detalle los mecanismos que subyacen en la decisión de acudir, hacen de ella una variable interesante desde una perspectiva de planificación de servicios.

En las últimas décadas se elaboraron varios modelos teóricos que profundizan en los aspectos económicos, psicosociales y de comportamiento que influyen en la utilización de los servicios sanitarios ${ }^{9}$. El más referenciado en la literatura es el modelo conductual de Andersen ${ }^{10}$, con modificaciones posteriores ${ }^{11,12}$, según el cual la utilización de servicios responde a una relación entre factores predisponentes, factores facilitadores y factores de necesidad.

Entre los factores predisponentes, una variable relevante es el sexo. De hecho, el patrón de consumo de servicios médicos es diferente entre las mujeres y los varones. En los países desarrollados, las mujeres expresan más síntomas, tienen una mayor prescripción de fármacos y producen un mayor número de consultas médicas ${ }^{13,14}$, lo cual parece independiente de las enfermedades basales ${ }^{15}$ y del que se ha sugerido que está relacionado con la percepción de su estado de salud ${ }^{16}$.

Las diferencias de sexo, a tenor de estas consideraciones, parecen tener un impacto importante en la relación entre la CVRS y el consumo de consultas. Desde un punto de vista metodológico, numerosos estudios dirigidos a estudiar dicha relación han considerado el sexo como una variable de ajuste ${ }^{17-21}$. Dentro de las escasas investigaciones que analizan por separado, en ambos sexos, la influencia de la CVRS sobre los diferentes patrones de utilización de servicios sanitarios se encuentra la de Redondo-Sendino et al ${ }^{22}$, que tiene la limitación de que los datos del estudio, incluido el consumo de servicios sanitarios, fueron reportados por los participantes mediante un cuestionario.

El objetivo del trabajo fue conocer la influencia de la CVRS en el consumo de consultas de atención primaria (AP) para las personas de 65 y más años, en un análisis diferenciado por sexo así como analizar su peso relativo frente a otras variables clínicas, demográficas, y de apoyo social.

\section{MATERIAL Y MÉTODOS}

La población de estudio fueron sujetos de 65 años y más, de ambos sexos, adscritos a dos cupos de Medicina de Familia de las Zonas Básicas de Salud (ZBS) de Camargo y Suances (Cantabria), ambas semiurbanas y demográficamente muy similares. 
El diseño fue transversal analítico, estratificado por sexo. El tamaño muestral se calculó conforme a los requisitos de la regresión lineal múltiple ${ }^{23}$, de acuerdo con las siguientes especificaciones: un error $\alpha=0,05$, una potencia del $85 \%$ para detectar un valor del coeficiente B de 0,15 y un error estándar $=0,5$. El tamaño muestral fue ampliado en un $10 \%$ en previsión de pérdidas de información, y quedó finalmente establecido en 346 sujetos. La muestra se repartió entre los cupos médicos de Camargo y Suances en 155 y 191 participantes, respectivamente, en proporción al número de sujetos $\geq 65$ años.

Mediante un muestreo consecutivo, cada paciente que acudió a consulta médica o de enfermería por cualquier motivo y cumplía los criterios de inclusión (edad $\geq 65$ años y pertenencia al cupo) era invitado a participar. Los pacientes fueron informados del objetivo del estudio y dieron su consentimiento por escrito. Fueron criterios de exclusión la situación de estar institucionalizado/a, la incapacidad para comunicarse adecuadamente con el evaluador (hipoacusia grave, disfasia) y un deterioro cognitivo moderado o grave, definido por 4 errores en el test de Pfeiffer (si había realizado estudios superiores), $\geq 5$ errores (si completó estudios primarios) o bien $\geq 6$ errores (si era analfabeto o no había realizado estudios primarios). Hubo 2 renuncias a participar. La inclusión de los participantes en el estudio se realizó entre los meses de febrero y diciembre de 2013.

La variable resultado era el número de visitas al Centro de Salud (VCS), definida como todos los contactos del paciente con los profesionales del equipo de AP, en el centro y en su domicilio durante el último año.

La CVRS fue evaluada mediante el cuestionario EuroQol-5D ${ }^{24}$ (EQ-5D), en su formato original de 3 niveles de respuesta para cada dimensión. Se trata de una herramienta genérica de medición de la CVRS, validada en nuestro país por Badia et $\mathrm{al}^{25}$, que consta de 3 partes: un sistema descriptivo que refleja el estado percibido de salud en
5 dimensiones (movilidad, cuidado personal, actividades cotidianas, dolor/malestar y ansiedad/depresión) clasificada cada una de ellas 3 niveles de gravedad (sin problemas, algunos o moderados problemas, y muchos o graves problemas, codificados como 1, 2 ó 3 , respectivamente). El resultado 1-1-1-1-1 corresponde a un estado óptimo. La segunda parte es una escala visual analógica (EVA) en forma de termómetro, cuyos extremos son 0 y 100 , el peor y el mejor estado de salud imaginable, respectivamente. El sujeto debe señalar el punto en la escala que indica su estado de salud en el momento en que se le encuesta ("en el día de hoy"). La tercera parte es el índice EQ-5D, una medida del valor social del estado de salud, que surge de la transformación de los 5 dígitos del sistema descriptivo en una puntuación. Se basa en las "utilidades" o preferencias de los sujetos respecto al estado de salud y tiene valores específicos para cada país.

El cuestionario EQ-5D fue utilizado para evaluar la CVRS en la Encuesta Nacional de Salud de España en su última edición de 2011-2012 ${ }^{26}$. Aun no habiendo sido disenado específicamente para AP y a pesar de su menor capacidad de discriminación en comparación con los instrumentos específicos, se propone como herramienta útil para evaluar la CVRS en el primer nivel de atención ${ }^{27}$.

En línea con otros autores ${ }^{28}$, se siguió una adaptación del modelo clásico de Andersen de predicción de consumo de servicios. Así, las variables explicativas se agruparon en 3 categorías: factores demográficos, factores de apoyo o entorno social e indicadores de salud. Las variables demográficas analizadas fueron el sexo, la edad, y el nivel de estudios. En el análisis del apoyo social se estudiaron la suficiencia de ingresos económicos, si se vive solo/a o no y el tiempo que se está solo/a. Por último, como variables clínicas se analizaron el peso, la talla, el índice de masa corporal (IMC), el deterioro cognitivo -test de Pfeiffer-, el grado de auto- 
nomía -índice de Barthel-, el grado de comorbilidad -índice de Charlson (iCh)-, el nivel de actividad física (AF), el consumo de tabaco y alcohol, y el uso de psicofármacos. Las definiciones precisas de las variables se detallaron en el protocolo del estudio. Mediante entrevista personal fueron cumplimentados el nivel de estudios, las variables de apoyo social, la AF, el consumo de tabaco y alcohol, el índice de Barthel, el test de Pfeiffer y el cuestionario EQ-5D. El resto de variables (edad, peso, talla, IMC, comorbilidad y VCS) se obtuvieron directamente de la historia clínica informatizada.

El estudio fue aprobado por el Comité Ético de Investigación Clínica de Cantabria. Se preservó adecuadamente el anonimato de los participantes.

Para el análisis descriptivo se calcularon el porcentaje y la media con la desviación estándar (DS) así como los terciles de las distribuciones: la edad, el índice EQ-5D y VCS. Se comprobó el ajuste a la normalidad y se recurrió a las pruebas de contraste $t$ de Student o U de Mann-Whitney para variables cuantitativas según siguieran la distribución normal o no, respectivamente, y chi ${ }^{2}$ para variables categóricas. Debido al escaso número de elementos en algunas categorías, las variables inicialmente politómicas fueron transformadas en dicotómicas. Así ocurrió con el nivel de estudios, la suficiencia de ingresos, si se vive solo o con compañía, si se pasa solo/a algún tiempo durante el día, la $\mathrm{AF}$, el consumo de tabaco y el de alcohol. Estas variables dicotómicas fueron utilizadas tanto en el análisis bivariante como en los 2 modelos multivariantes.

Considerando la variable dependiente VCS en forma categórica (dicotomizada según la mediana de la distribución), se realizaron 2 análisis: el cálculo de la relación global entre la CVRS (estado subóptimo vs estado óptimo) y VCS, mediante la odds ratio (OR) de prevalencia, cruda y ajustada sucesivamente por las variables demográficas, por las variables clínicas y por las variables de apoyo social. Asimismo, mediante tablas de contingencia fueron calculadas las OR de prevalencia para las variables independientes y sus intervalos de confianza (IC) al 95\%.

En una segunda fase, una vez descartadas las variables no asociadas significativamente con VCS en el análisis crudo, y con el sexo como factor de estratificación, se elaboraron 2 modelos de regresión lineal múltiple con las 10 variables restantes, mediante el procedimiento Enter y VCS, en este caso en expresión cuantitativa. Basándonos en la comparabilidad de los coeficientes $\beta$ estandarizados, sus valores fueron ponderados mediante el cálculo del porcentaje que suponía cada coeficiente $\beta$ respecto de la suma de todos los coeficientes $\beta$ en valor absoluto. Ambos modelos se validaron mediante la verificación de los supuestos de la regresión lineal ${ }^{29}$.

El índice EQ-5D con valores para la población española se obtuvo mediante un software gratuito patrocinado por la Universidad de Bristol, disponible en Internet ${ }^{30}$. Los cálculos fueron realizados con SPSS ${ }^{\circ}$ V.15 (Chicago, Illinois, EEUU) considerándose estadísticamente significativa una $\mathrm{p}<0,05$.

\section{RESULTADOS}

La edad media de las 346 personas participantes en el estudio (191 mujeres y 155 varones) fue de 76 años. Con mayor frecuencia que los varones, las mujeres vivían solas $[39(20 \%)$ frente a $14(9 \%)]$, estaban más tiempo solas $[22(11 \%)$ frente a $4(2 \%)]$, el nivel de estudios era menor [28(15\%) sin estudios, frente a 7(4\%)], tenían un menor grado de actividad física [36(18\%) eran sedentarias, frente a $23(14 \%)$ ], no consumían tabaco ni alcohol pero sí más psicofármacos $[74(39 \%)$ frente a $33(21 \%)$ ] y cometieron más errores en el test de Pfeiffer (media de 1 frente a 0,4) (tabla 1). Los varones presentaron un mayor grado de comorbilidad (índice de Charlson de 1,2 frente a 0,7$)$ y mejores indicadores de apoyo social. El índice de Barthel fue de 98 puntos en ambos casos.Las mujeres valoraron su CVRS peor que los varones (tabla 2), ob- 


\begin{tabular}{|c|c|c|c|c|}
\hline \multicolumn{5}{|c|}{$\begin{array}{c}\text { Tabla } 1 \\
\text { Características de la muestra (I): Variables antropométricas, sociodemográficas } \\
\text { y clínicas }\end{array}$} \\
\hline Variable & Total muestra & $\begin{array}{c}\text { Mujeres } \\
\mathrm{n}=191\end{array}$ & $\begin{array}{c}\text { Varones } \\
\mathrm{n}=155\end{array}$ & $\mathrm{p}$ \\
\hline Edad (años)* & $73(6)$ & $73(6)$ & $72(6)$ & NS \\
\hline Peso $(\mathrm{kg})^{*}$ & $74(11)$ & $70(10)$ & $78(10)$ & 0,0001 \\
\hline Talla $(\mathrm{cm})$ & $160(8)$ & $155(6)$ & $166(5)$ & 0,0001 \\
\hline $\operatorname{IMC}\left(\mathrm{Kg} / \mathrm{m}^{2}\right)^{*}$ & $28(4)$ & $29(4)$ & $28(4)$ & NS \\
\hline Sin estudios & $35(10)$ & $28(15)$ & $7(4)$ & \multirow{4}{*}{0,0001} \\
\hline Estudios primarios & $249(72)$ & $142(75)$ & $107(69)$ & \\
\hline Estudios secundarios & $46(13)$ & $18(9)$ & $28(18)$ & \\
\hline Estudios universitarios & $16(4)$ & $3(1)$ & $13(8)$ & \\
\hline Ingresos insuficientes & $98(28)$ & $61(32)$ & $37(24)$ & \multirow{3}{*}{ ns } \\
\hline Ingresos justos & $189(54)$ & $96(50)$ & $93(60)$ & \\
\hline Ingresos holgados & $59(17)$ & $34(17)$ & $25(16)$ & \\
\hline Vive solo/a & $53(15))$ & $39(20)$ & $14(9)$ & \multirow{4}{*}{0,0001} \\
\hline Vive con la pareja & $136(39)$ & $63(33)$ & $73(47)$ & \\
\hline Vive con la familia & $74(21)$ & $60(31)$ & $14(9)$ & \\
\hline Vive con la pareja y la familia & $82(23)$ & $29(15)$ & $53(34)$ & \\
\hline Pasa solo/a todo el día & $26(7)$ & $22(11)$ & $4(2)$ & \multirow{4}{*}{0,01} \\
\hline Pasa solo/medio día & $92(26)$ & $54(28)$ & $38(24)$ & \\
\hline Está solo/a a ratos & $159(46)$ & $80(42)$ & $79(51)$ & \\
\hline Nunca está solo & $67(19)$ & $35(18)$ & $32(21)$ & \\
\hline Test de Pfeiffer ( $n^{\circ}$ de errores)* & $0,8(1)$ & $1(1)$ & $0,4(1)$ & 0,0001 \\
\hline Indice de Barthel* & $98(5)$ & $98(4)$ & $98(3)$ & $\mathrm{ns}$ \\
\hline Indice de Charlson* & $0,9(1)$ & $0,7(1)$ & $1,2(1)$ & 0,0001 \\
\hline Sedentarismo & $59(17)$ & $36(18)$ & $23(14)$ & \multirow{3}{*}{0,0001} \\
\hline Actividad física ligera & $203(58)$ & $126(66)$ & $77(49)$ & \\
\hline Actividad física moderada/intensa & $84(24)$ & $29(15)$ & $55(35)$ & \\
\hline No fumador & $187(54)$ & $150(78)$ & $37(24)$ & \multirow{3}{*}{0,0001} \\
\hline Ex fumador/a & $122(35)$ & $27(14)$ & $95(61)$ & \\
\hline Fumador/a actual & $37(10)$ & $14(7)$ & $23(14)$ & \\
\hline No consumo de alcohol & $186(53)$ & $147(77)$ & $39(25)$ & \multirow{3}{*}{0,0001} \\
\hline Consumo leve/moderado & $105(44)$ & $44(23)$ & $109(70)$ & \\
\hline Consumo excesivo de alcohol & $7(0,2)$ & & $7(4)$ & \\
\hline Consumo de psicofármacos & $107(31)$ & $74(39)$ & $33(21)$ & 0,001 \\
\hline $\begin{array}{l}\text { IMC: índice de masa corporal. ns: } \\
\text { Las variables cuantitativas edad, } p \\
\text { expresadas como media (desviació } \\
\text { test U de Mann-Whitney }(*) \text { y test }\end{array}$ & $\begin{array}{l}\text { cativo/a. } \\
\text { IMC, test } \\
\text { ir). El resto d } \\
\text { ent (=). En } 1\end{array}$ & $\begin{array}{l}\text { indice de } \\
\text { categóric } \\
\text { categóri }\end{array}$ & $\begin{array}{l}\text { dice de } \\
\text { \%). Pue } \\
\text { de cont }\end{array}$ & $\begin{array}{l}\text { son, están } \\
\text { contraste } \\
\text { ue } \text { chi }^{2} \text {. }\end{array}$ \\
\hline
\end{tabular}

jetivándose en 4 dimensiones del cuestionario, en la EVA (64 frente a 69) y en el índice EQ-5D $(0,82$ frente a 0,88$)$.

El estado óptimo (1-1-1-1-1) fue referido por $45(23 \%)$ mujeres y $72(46 \%)$ varones $(p=0,0001)$, con un empeoramiento progresivo del índice EQ-5D a medida que avanzaba la edad. La variable VCS no mostró diferencias estadísticamente significativas por sexo: mientras que los varones presentaron una media de 25 visitas, las mujeres visitaron el CS una media de 23 veces $(p=0,12)$. En ambos sexos, la variable VCS se correla- cionó de forma positiva con la edad, con un valor Rho-Spearman de 0,19 en las mujeres $y$ de 0,35 en los varones $(p<0,05$ en ambos casos)

Un bajo nivel de estudios se asoció, respecto a haber realizado estudios superiores, a un mayor consumo de consultas: los valores de OR fueron de 7,1 (IC95\%: 2-25) para las mujeres y de 3,4 (IC95\%:1,5-7,4) para los hombres. Se observaron diferencias con significación estadística entre ambas localidades respecto a la CVRS, con un índice EQ-5D de 0,87(0,1) en Suances y 
Tabla 2

Características de la muestra (II):

Calidad de Vida Relacionada con la Salud y Visitas al Centro de Salud

\begin{tabular}{|c|c|c|c|c|}
\hline & $\begin{array}{c}\text { Total } \\
\text { muestra }\end{array}$ & $\begin{array}{c}\text { Mujeres } \\
n=191\end{array}$ & $\begin{array}{c}\text { Varones } \\
\mathrm{n}=155\end{array}$ & $\mathrm{p}$ \\
\hline \multicolumn{5}{|l|}{ Movilidad } \\
\hline 1. No tengo problemas para caminar & $256(73)$ & $133(69)$ & $123(79)$ & \multirow{3}{*}{0,04} \\
\hline 2. Tengo algunos problemas para caminar & $90(26)$ & $58(30)$ & $32(20)$ & \\
\hline 3. Tengo que estar en la cama & - & - & - & \\
\hline \multicolumn{5}{|l|}{ Cuidado personal } \\
\hline 1. No tengo problemas con el cuidado personal & $318(91)$ & $175(91)$ & $143(92)$ & \multirow{3}{*}{ ns } \\
\hline 2. Tengo algunos problemas para lavarme o vestirme & $27(7)$ & $15(8)$ & $12(7)$ & \\
\hline 3. Soy incapaz de lavarme o vestirme & $1(0,2)$ & $1(0,5)$ & - & \\
\hline \multicolumn{5}{|l|}{ Actividades cotidianas } \\
\hline 1. No tengo problemas para realizar mis actividades cotidianas & $293(84)$ & $153(80)$ & $140(90)$ & \multirow{3}{*}{0,02} \\
\hline 2. Tengo algunos problemas para realizar mis actividades cotidianas & $49(14)$ & $36(18)$ & $13(8)$ & \\
\hline 3. Soy incapaz de realizar mis actividades cotidianas & $4(1,1)$ & $2(1)$ & $2(1)$ & \\
\hline \multicolumn{5}{|l|}{ Dolor/malestar } \\
\hline 1. No tengo dolor ni malestar & $169(48)$ & $77(40)$ & $92(59)$ & \multirow{3}{*}{0,002} \\
\hline 2. Tengo moderado dolor o malestar & $164(47)$ & $106\left(55^{\prime}\right)$ & $58(37)$ & \\
\hline 3. Tengo mucho dolor o malestar & $13(3,7)$ & $8(4)$ & $5(3)$ & \\
\hline \multicolumn{5}{|l|}{ Ansiedad/depresión } \\
\hline 1. No estoy ansioso ni deprimido & $257(74)$ & $127(66)$ & $130(84)$ & \multirow{3}{*}{0,001} \\
\hline 2. Estoy moderadamente ansioso o deprimido & $84(24)$ & $61(32)$ & $23(14)$ & \\
\hline 3. Estoy muy ansioso o deprimido & $5(1,4)$ & $3(1)$ & $2(1)$ & \\
\hline Escala Visual Analógica & $66(19)$ & $64(19)$ & $69(18)$ & 0,006 \\
\hline Indice EuroQol-5D & $0,84(0,2)$ & $0,82(0,2$ & $0,88(0,2)$ & \\
\hline Visitas al Centro de Salud & $24(19)$ & $23(19)$ & $25(20)$ & ns \\
\hline $65-70$ años & $19(17)$ & $19(15)$ & $20(19)$ & ns \\
\hline 71-76 años & $25(21)$ & $25(22)$ & $26(19)$ & ns \\
\hline$\geq 77$ años & $30(20)$ & $27(21)$ & $33(20)$ & 0,027 \\
\hline Indice EQ-5D $\leq 0,83$ & $30(23)$ & $29(23)$ & $30(23)$ & ns \\
\hline Indice EQ-5D, 0,84-0,91 & $21(17)$ & $16(11)$ & $28(20)$ & 0,002 \\
\hline Indice EQ-5D $\geq 0,92$ & $22(19)$ & $20(16)$ & $23(21)$ & ns \\
\hline
\end{tabular}

de $0,81(0,2)$ en Camargo ( $p=0,01)$. Tanto las mujeres como los varones de Suances puntuaron mejor su CVRS que sus homólogos de Camargo. Al profundizar en este resultado, hemos comprobado sobre nuestros datos que en un análisis de regresión lineal con el índice EQ-5D como variable dependiente, el factor localidad mantenía una asociación significativa con la CVRS tras ajustar por sexo, la edad, la comorbilidad, el nivel de estudios y el consumo de psicofármacos $(\beta=0,14 ; \mathrm{p}=0,007)$.

La CVRS se correlacionó de forma negativa con VCS (tabla 2), con un valor RhoSpearman de $-0,22(p=0,0001)$. A medida que se incrementaba el valor del índice EQ-5D descendía la media de consultas. En concreto, tomando como referencia el primer tercil, en el último del índice EQ-5D hubo un descenso en la media de consultas del $31 \%$ en las mujeres y del $23 \%$ en los varones. La asociación cruda entre la CVRS y VCS presentó una OR de prevalencia de 1,85 (1,2-2,9). Esta asociación se mantuvo estadísticamente significativa al ajustar por separado por las variables demográficas [OR=1,99 (IC95\%:1,2-3,2)], las variables clínicas $[\mathrm{OR}=1,79$ (IC95\%:1,1$2,9)]$ o bien las de apoyo social $[\mathrm{OR}=1,83$ (IC95\%:1,1-2,9)] (datos no mostrados). La tabla 3 muestra el grado de asociación no ajustada de las variables del estudio, respecto al consumo de consultas.

En el análisis multivariante, la relación de la CVRS con VCS fue de diferente magnitud según el sexo (tabla 4): mientras que en las mujeres, la CVRS mostró un coeficiente $\beta$ de 0,22 (el más elevado de las 10 variables 


\begin{tabular}{|c|c|c|c|}
\hline \multicolumn{4}{|c|}{$\begin{array}{c}\text { Tabla } 3 \\
\text { Variables del estudio y asociación no ajustada con el consumo de consultas }\end{array}$} \\
\hline \multirow[t]{2}{*}{ Variable } & \multicolumn{3}{|c|}{ Consumo de consultas } \\
\hline & $\mathrm{OR}^{\psi}$ & IC $95 \%$ & $\mathrm{p}$ \\
\hline Sexo (mujer) & 0,95 & $0,6-1,4$ & 0,82 \\
\hline Edad ( $\geq 73$ años $)$ & 2,27 & $1,3-3,5$ & 0,0001 \\
\hline Nivel de estudios (Sin estudios o estudios primarios) & 3,98 & $2,1-7,4$ & 0,0001 \\
\hline Suficiencia de ingresos (Insuficientes o justos) & 1,24 & $0,7-2,2$ & 0,44 \\
\hline Vive con... (Vive solo/a) & 1,23 & $0,6-2,2$ & 0,48 \\
\hline Pasa solo/a... (Todo el día) & 1,16 & $0,5-2,6$ & 0,70 \\
\hline Indice de masa corporal $\left(\geq 28,4 \mathrm{~kg} / \mathrm{m}^{2}\right)$ & 1,66 & $1,1-2,5$ & 0,02 \\
\hline Nivel cognitivo (Test Pfeiffer $>2$ errores) & 2,22 & $1,01-4,8$ & 0,046 \\
\hline Grado de autonomía (Indice de Barthel $\neq 100$ ) & 2,25 & $1,1-4,4$ & 0,02 \\
\hline Comorbilidad (Indice de Charlson $\neq 0$ ) & 3,31 & $2,1-5,1$ & 0,0001 \\
\hline Actividad física (Sedentarismo o AF ligera) & 1,80 & $1,1-2,9$ & 0,02 \\
\hline Consumo de tabaco & 1,10 & $0,7-1,6$ & 0,66 \\
\hline Consumo de alcohol & 0,61 & $0,4-0,9$ & 0,02 \\
\hline Consumo de psicofármacos & 2,18 & $1,3-3,5$ & 0,0001 \\
\hline CVRS (Estado subóptimo*) & 1,85 & $1,2-2,9$ & 0,007 \\
\hline
\end{tabular}

\begin{tabular}{|l|c|c|c|c|r|r|}
\hline \multicolumn{7}{|c|}{ Modelos de regresión lineal para la variable "visitas al centro de salud" } \\
\hline \multirow{3}{*}{ Variables explicativas } & \multicolumn{3}{|c|}{$\begin{array}{c}\text { Modelo } 1 \\
\text { Mujeres }\end{array}$} & \multicolumn{3}{c|}{$\begin{array}{c}\text { Modelo } 2 \\
\text { Varones }\end{array}$} \\
\cline { 2 - 8 } & $\begin{array}{c}\text { Coeficiente } \\
\beta^{*}\end{array}$ & IC-95\% & $\mathrm{p}$ & $\begin{array}{c}\text { Coeficiente } \\
\beta^{*}\end{array}$ & IC-95\% & $\mathrm{p}$ \\
\hline Edad & 0,11 & $-1-0,8$ & 0,12 & 0,16 & $-0,01-1,07$ & 0,055 \\
\hline Nivel de estudios & 0,16 & $-18-1,3$ & 0,02 & 0,06 & $-9,8-4,2$ & 0,43 \\
\hline Indice de masa corporal & 0,10 & $-0,2-1$ & 0,20 & 0,03 & $-0,7-1,1$ & 0,65 \\
\hline Grado de autonomía & 0,02 & $-0,7-0,5$ & 0,75 & 0,06 & $-0,8-0,4$ & 0,52 \\
\hline Comorbilidad & 0,19 & $0,8-6,7$ & 0,01 & 0,34 & $2,7-7,2$ & 0,0001 \\
\hline Actividad física & 0,02 & $-8,4-6,6$ & 0,81 & 0,06 & $-9,7-4,2$ & 0,43 \\
\hline Consumo de psicofármacos & 0,13 & $-10,3-0,2$ & 0,06 & 0,01 & $-8,1-7,2$ & 0,91 \\
\hline CVRS & 0,22 & $-36,7--6,9$ & 0,004 & 0,03 & $-15,6-23,1$ & 0,70 \\
\hline Consumo de alcohol & 0,04 & $-8-4,5$ & 0,58 & 0,16 & $-0,1-15,1$ & 0,052 \\
\hline Nivel cognitivo & 0,02 & $-2-2,9$ & 0,72 & 0,01 & $-4-3,6$ & 0,91 \\
\hline CVRS: Calidad de vida relacionada con la salud. IC: Intervalo de confianza. * En valor absoluto. & \\
\hline
\end{tabular}

del modelo) en los varones el coeficiente fue prácticamente nulo $(\beta=0,03)$. Esos valores de $\beta$, expresados en porcentaje sobre la varianza explicada por el modelo, señalaban que la CVRS aportaba en las mujeres el $22 \%$ de la capacidad explicativa global, mientras que en los varones, tan sólo suponía el 3\% (figura 1). El valor del coeficiente de determinación $\left(\mathrm{R}^{2}\right)$ fue 0,23 en ambos modelos.
Los modelos fueron validados mediante la comprobación de los supuestos de la regresión lineal. Las curvas superpuestas a los histogramas de residuos tipificados se aproximaron a la curva normal tipificada $\mathrm{N}(0,1)$, con una media (DS) de $0(0,97)$ y $0(0,96)$. Unos valores del estadístico Durbin-Watson situados entre 1,98 y 1,79 confirmaron la independencia de los residuos. Por último, se 


\section{Figura 1}

\section{Capacidad explicativa de la CVRS respecto al consumo de consultas en ambos sexos}

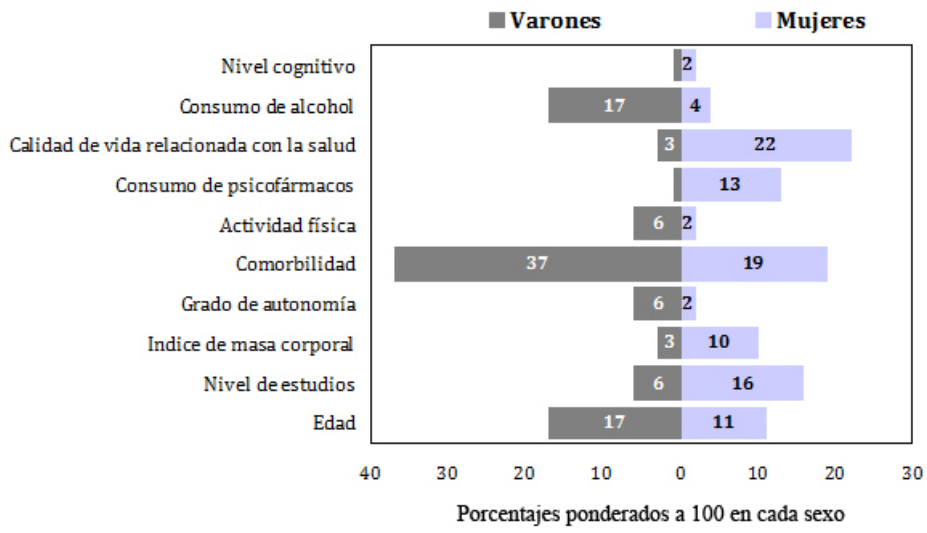

demostró la ausencia de multicolinealidad, pues ninguna de las correlaciones lineales superaba el valor convencional de $0,50 \mathrm{y}$ el factor de inflación de la varianza fue inferior a 2 en todos los casos.

\section{DISCUSIÓN}

Nuestra investigación muestra, por un lado, la peor valoración que hacen las mujeres de su CVRS. Estos resultados, que afectan tanto a las diferentes dimensiones del sistema descriptivo del EQ-5D como a la EVA, fueron similares a los observados en otros estudios realizados sobre población española no institucionalizada de $>65$ años $^{31-33}$. De hecho, en concordancia con el valor 66 de la EVA observado en nuestro estudio, se han reportado valores de $68^{31}, 66^{32}$ o $70^{33}$.

Por otro lado, aunque algunos autores sostienen que la CVRS por sí sola es una débil variable explicativa de sobreutilización de consultas ${ }^{17}$, se observa asimismo la consistente asociación negativa que existe entre la CVRS y el consumo de recursos observada con anterioridad ${ }^{5,34}$. En este sentido, hemos comprobado que a medida que aumenta la valoración de la CVRS se reduce el número de VCS en ambos sexos.
Como dato de interés, se observan notables diferencias entre ambos sexos respecto a la influencia de la CVRS en la utilización de servicios. Mientras que la CVRS en las mujeres se muestra como el factor más determinante de consumo de consultas, por delante e independientemente de la comorbilidad, el nivel de estudios, la edad y el resto de variables analizadas, por el contrario, la CVRS tiene una influencia prácticamente nula en los varones.

Este resultado es concordante con lo publicado. En un estudio longitudinal sobre 509 pacientes, Bertakis et $\mathrm{al}^{21}$ observaron que las mujeres presentaban una peor CVRS evaluada mediante el cuestionario SF-36, un menor nivel educativo, un mayor número de visitas al primer nivel asistencial y un mayor número de pruebas diagnósticas. Mediante ecuaciones de regresión investigaron la relación entre el sexo y los costes médicos, controlando por diferentes variables de confusión. En el caso del primer nivel asistencial demostraron que la edad $(\beta=0,20)$, la salud física percibida $(\beta=0,10)$ y el sexo femenino $(\beta=0,14)$ eran predictores significativos independientes de un mayor gasto sanitario. En opinión de los autores, la peor percepción de salud en las mujeres genera una serie de cuidados más costosos y complicados 
que en los varones, los cuales presentaron una mejor salud percibida basal.

En la misma línea, Redondo-Sendino et $\mathrm{al}^{22}$, mediante un estudio transversal sobre 3.030 sujetos no institucionalizados mayores de 60 años, observaron que el mayor nivel de utilización de servicios médicos por parte de las mujeres se asociaba significativamente a la CVRS. Concretamente, a partir de una OR cruda de 1,44 , la asociación descendía a un valor de 1,09 al ajustar por la CVRS y a un valor de 1,00 al ajustar simultáneamente por la CVRS y por el número de enfermedades crónicas. La atenuación de la diferencia al controlar estos dos factores llevó a los autores a sugerir que la CVRS y las enfermedades crónicas son las variables más explicativas de la mayor utilización de servicios sanitarios por parte de las mujeres mayores de 60 años.

La fuerte relación que se observa en las mujeres entre la CVRS y el uso de servicios sanitarios puede ser interpretada desde el análisis de las diferencias por sexo respecto a la salud. Se han propuesto varias hipótesis explicativas $^{35}$ y una de ellas es la teoría de la necesidad diferencial, que se basa en que las mujeres tienen una mayor necesidad objetiva de servicios de salud. Así, se ha descrito que son más sensibles al malestar ${ }^{15}$ y tienen una mayor exposición a problemas emocionales ${ }^{36}$. Además, en términos generales son más sedentarias que los varones, tienen menor movilidad y presentan más dolor ${ }^{37}$. Una segunda teoría es la de la socialización de género, basada en que la socialización diferencial de mujeres y hombres influye sobre sus actitudes y conductas de búsqueda de atención de salud ${ }^{35}$. Explicaría el hecho de que los hombres tienden a ver la enfermedad y la búsqueda de atención médica como una manifestación de debilidad ${ }^{38}$, el que las mujeres sean más proclives a solicitar ayuda médica y la mejor aceptación social de sus quejas ${ }^{16}$.

El clásico paradigma "las mujeres están más enfermas, pero los varones fallecen antes" puede tener su origen en un tipo de morbilidad muy influida por factores psico- sociales y que habitualmente no es causa de muerte $^{13,16}$. En concreto, se ha señalado que el mayor consumo de servicios médicos y de fármacos observado en las mujeres estaría relacionado con el hecho de que sobre ciertas patologías agudas y crónicas, como la artrosis, los factores sociales y psicológicos actúan incrementando la diferencia entre géneros ${ }^{16}$. Asimismo, hay que considerar que existen diferencias en la práctica asistencial según el sexo del paciente ${ }^{15}$.

La comorbilidad fue significativamente mayor en los varones, con un 37\% de la capacidad explicativa del modelo, el principal predictor de consumo de consultas en este grupo. En nuestra opinión este resultado puede ser explicado, al menos en parte, por las diferencias de sexo en salud y por la herramienta seleccionada para su valoración, el $\mathrm{iCh}^{39}$.

Tradicionalmente, en los varones se han reportado una mayor prevalencia de enfermedades graves y mayores tasas de mortalidad y de hospitalización ${ }^{16,21}$ y se ha sugerido ${ }^{40}$ que las diferentes tasas de hospitalización son debidas a un tipo de patología (cardiovascular y respiratoria) más grave, que suelen padecer los varones, frente a las patologías que presentan las mujeres ${ }^{40}$. Sin embargo, también se ha invocado una práctica asistencial sesgada, en el sentido de que una paciente con patología cardiovascular es derivada con menor frecuencia al nivel especializado que un paciente varón ${ }^{15}$. Por otro lado, una de las limitaciones del iCh como medida de comorbilidad es que sólo contempla enfermedades graves potencialmente mortales. Además, la morbilidad cardiovascular tiene un elevado protagonismo en su sistema de puntuación ${ }^{41}$. En consecuencia, el iCh refleja un tipo de patología tradicionalmente más frecuente en los varones. Cabe conjeturar que una medida de la comorbilidad que incluyese patologías como la artrosis y los trastornos ansioso-depresivos, de bajo riesgo de mortalidad, alta prevalencia en las mujeres y con un impacto relevante sobre la autonomía, la CVRS y el 
consumo de consultas, podría teóricamente incrementar en las mujeres la capacidad predictiva de esta variable respecto al uso de servicios $^{42-44}$.

Un bajo nivel de estudios es asimismo un factor estadísticamente asociado e independientemente a un mayor consumo de consultas de AP, tal y como se ha observado en estudios previos ${ }^{9,19,34}$ y un alto nivel educativo se ha relacionado con un menor número de consultas, ya sea debido a un mejor estado de salud autopercibido u objetivado ${ }^{45}$. De igual forma, en nuestra investigación el bajo nivel de estudios se asoció a un mayor consumo de consultas. Sin embargo, estos resultados son discordantes respecto a lo observado por Rodríguez et $\mathrm{al}^{46}$ en un análisis realizado sobre datos de la Encuesta Nacional de Salud de 1997, en el que observaron que el consumo de servicios por parte de las mujeres era similar para cualquier nivel de estudios, al contrario de lo que ocurría en los varones, en los que sí se apreciaba un mayor número de consultas en el grupo con bajo nivel educativo. Dicho estudio no aportaba regresiones múltiples $\mathrm{y}$, según nuestros resultados, tras ajustar por variables de confusión, un bajo nivel de estudios es el tercer factor más explicativo de VCS en las mujeres.

Cabe añadir asimismo una reflexión acerca de la posible influencia del factor geográfico o de localidad en la CVRS. Los sujetos participantes correspondían a dos ZBS cuyas cabeceras de municipio distan $30 \mathrm{~km}$. Además, ambas son semiurbanas, con un grado similar de dispersión geográfica. La estructura poblacional y la cobertura universal de la asistencia pública son otras características que las hacen muy similares. Sin embargo, a pesar de estas similitudes estructurales, se observan diferencias estadísticamente significativas entre ambas localidades en cuanto a la CVRS y una asociación del factor localidad con la CVRS que mantenía la significación estadística tras ajustar por variables de confusión. En este sentido, desde hace tiempo se ha sugerido la posible influencia de la localidad en la que se realizan los estudios de $\mathrm{CVRS}^{47} \mathrm{y}$, de hecho, en nuestro país se observan diferencias en la CVRS entre regiones y dentro de la misma región ${ }^{48}$. El factor geográfico o de localidad influye sobre la salud y sobre la calidad de vida de los individuos ${ }^{49}$ mediante unas variables interpuestas de difícil ajuste, como puede ser el sentimiento de comunidad ${ }^{50}$. Por lo tanto, la localidad en la que el individuo desarrolla su vida cotidiana puede ser un factor para considerar en los estudios sobre CVRS y, desde un punto de vista metodológico, puede valorarse su utilidad como variable de ajuste. Sin embargo, en nuestro estudio se decidió no incluirla en los modelos multivariantes por el riesgo de producir un sobreajuste que potencialmente podría disminuir la precisión de las estimaciones.

Nuestro estudio presenta varias limitaciones. Una de ellas es su diseño transversal, que dificulta establecer la direccionalidad de las asociaciones. Otra es el ámbito local, pues no permite extrapolar los resultados a otras poblaciones. Asimismo, cabe señalar que los dos modelos multivariantes fueron elaborados tras distribuirse la muestra calculada inicialmente, en mujeres y hombres según acudían a consulta. Aunque ello no afecta a las asociaciones observadas, hay que interpretar cuidadosamente los resultados no significativos que se han observado en ambos modelos, debido a la previsible pérdida de potencia estadística.

Por otro lado, los autores entendemos que el principal interés del estudio es la aportación de un análisis estratificado por sexo. Hemos comprobado que en un hipotético modelo de regresión sobre la muestra global, sin estratificar por sexo y que lo incluyese como una variable de ajuste más, el factor más determinante de VCS pasa a ser la comorbilidad seguida de la edad y la CVRS. A tenor de este resultado, el análisis por estratos ha permitido conocer unas diferencias entre ambos sexos que de otra forma permanecerían ocultas. 
En nuestra opinión, las conclusiones de este estudio tienen una doble utilidad. Desde la perspectiva de la planificación sanitaria, es de gran interés conocer cuáles son los determinantes principales en la decisión de cada persona de acudir a una consulta médica. Al igual que en otros estudios que han trabajado en esta línea ${ }^{20}$, entendemos que los resultados observados pueden contribuir a identificar las características de los pacientes que utilizan más frecuentemente las consultas de AP. Por otro lado, ofrecen información para el análisis de la equidad en el acceso a los servicios de salud, en el que la consideración de los factores de género se muestra ciertamente relevante ${ }^{35}$.

Futuras investigaciones que aporten una evidencia más sólida pueden confirmar nuestras conclusiones, es decir, la CVRS como un determinante principal e independiente de consumo de consultas en AP para la mujer de 65 y más años, mientras que en los varones de la misma edad la influencia de la CVRS sobre el consumo de consultas es prácticamente nula.

\section{BIBLIOGRAFÍA}

1. Schwartzmann L. Calidad de vida relacionada con la salud: aspectos conceptuales. Ciencia y enfermería. 2003;9:9-21.

2. Patrick D. Erickson P. Health Policy, Quality of Life: Health Care Evaluation and Resource Allocation. Oxford University Press. New York;1993.

3. Ruiz de Velasco I, Quintana JM, Padiema JA, Aróstegui I, Bernal A, Pérez-Izquierdo J et al. Validez del Cuestionario de calidad de vida SF-36 como indicador de resultados de procedimientos médicos y quirúrgicos. Rev Calidad Asistencial. 2002;17:206-12.

4. Miilunpalo S, Vuori I, Oja P, Pasanen M, Urponen H. Self-rated health status as a health measure: the predictive value of self-reported health status on the use of physician services and on mortality in the working-age population. J Clin Epidemiol. 1997;50:51728.

5. Connelly JE, Philbrick JT, Smith GR Jr, Kaiser DL, Wymer A. Health perceptions of primary care patients and the influence on health care utilization. Med Care. 1989;27:S99-109.
6. König HH, Heider D, Lehnert T, Riedel-Heller SG, Angermeyer MC, Matschinger $\mathrm{H}$ et al. Health status of the advanced elderly in six European countries: results from a representative survey using EQ-5D and SF-12. Health Qual Life Outcomes. 2010;8:143.

7. Azpiazu-Garrido M, Cruz-Jentoft A, Villagrassa-Ferrer JR, Abanades-Herranz JC, García-Marín N, Alvear-Valero de Bernabé F. Factores asociados a mal estado de salud percibido o a mala calidad de vida en personas mayores de 65 años. Rev Esp Salud Pública. 2002;76:683-99.

8. Ministerio de Sanidad, Servicios Sociales e Igualdad. Encuesta Nacional de Salud 2011-2012.

9. Hulka BS, Wheat JR. Patterns of utilization. The patient perspective. Med Care. 1985;23:438-60.

10. Andersen RM. Revisiting the behavioral model and access to medical care: Does it matter? Journal of Health and Social Behavior. 1995;36:1-10.

11. Andersen R, Newman JF. Societal and Individual Determinants of Medical Care Utilization in the United States. The Milbank Quarterly. 2005;83(4):1-28.

12. Rebhan P. Health Care Utilization: Understanding and applying theories and models of health care seeking behavior. Case Western Reserve University, 2011. Disponible en: http://www.cwru.edu/med/epidbio/mphp439/ healthcareutil.pdf

13. Macintyre S, Hunt K, Sweeting H. Gender differences in health: Are things really as simple as they seem? Soc Sci Med. 1996;42:617-24.

14. Scaife B, Gill P, Heywood P, Neal R. Socio-economic characteristics of adult frequent attenders in general practice: secondary analysis. Fam Pract. 2000;17:298-304.

15. Suominen-Taipale AL, Martelin T, Koskinen S, Holmen J, Johnsen R. Gender differences in health care use among the elderly population in areas of Norway and Finland. A cross-sectional analysis based on the HUNT study and the FINRISK Senior Survey. BMC Health Serv Res. 2006;6:110.

16. Verbrugge LM. Sex differentials in health. Public Health Reports. 1982;97:417-37.

17. Jordan K, Ong BN, Croft P. Previous consultation and self reported health status as predictors of future demand for primary care. J Epidemiol Community Health. 2003;57:109-13.

18. Little P, Somerville J, Williamson I, Warner J, Moore $\mathrm{M}$, Wiles R et al. Psychosocial, lifestyle and health status variables in predicting high attendance among adults. $\mathrm{Br}$ J Gen Pract. 2001;51:987-94.

19. Fernández-Olano C, López-Torres Hidalgo JD, Cerdá-Díaz R, Requena-Gallego M, Sánchez-Castaño C, Urbistondo-Cascales $\mathrm{L}$ et al. Factors associated with health care utilization by the elderly in a public health system. Health Policy. 2006;75:131-9. 
20. Martín-Fernández J, Gómez-Gascón T, del CuraGonzález MI, Tomás-García N, Vargas-Machuca C, Rodríguez-Martínez C. La calidad de vida relacionada con la salud como factor explicativo de la utilización de la consulta de medicina de familia: un estudio bajo el modelo conductual. Rev Esp Salud Pública. 2010;84:309-19.

21. Bertakis KD, Azari R, Helms LJ, Callahan EJ, Robbins JA. Gender differences in the utilization of health care services. J Fam Pract. 2000;49:147-52.

22. Redondo-Sendino A, Guallar-Castillón P, Banegas JR, Rodríguez-Artalejo F. Gender differences in the utilization of health-care services among the older adult population of Spain. BMC Public Health. 2006;6:155.

23. Dupont WD, Plummer WD. Power and sample size calculations for studies involving linear regression. Controlled Clinical Trials. 1998;19:589-601.

\section{EuroQol website: http://www.euroqol.org/}

25. Badia X, Roset M, Montserrat S, Herdman M, Segura A. La versión española del EuroQol: descripción y aplicaciones. Med Clin (Barc). 1999;112 (Supl 1):7986.

26. Ministerio de Sanidad, Servicios Sociales e Igualdad. Encuesta Nacional de Salud 2011-2012. Disponible en:http://www.ine. es $/$ jaxi/menu.do?type $=$ pcaxis $\&$ path $=\% 2 \mathrm{Ft} 15 /$ p419\&file=inebase $\& \mathrm{~L}=0$ ]

27. Herdman M, Badia X, Berra S. El EuroQol-5D: una alternativa sencilla para la medición de la calidad de vida relacionada con la salud en atención primaria. Aten Primaria. 2001;28(6):425-30.

28. Díez-Ticio A, Berdún P. Envejecimiento y utilización de servicios sanitarios: Un análisis de sus determinantes en la ciudad de Zaragoza. Ponencia al XVI Encuentro de Economía Pública. Granada, 5-6 febrero de 2009. Disponible en: http://dialnet.unirioja.es/servlet $/$ libro? codigo $=360583$

29. Ballance DL. Assumptions in multiple regression: A tutorial 2011. Disponible en: http://www.dianneballanceportfolio.com/uploads/1/2/8/2/12825938/assumptions_in_multiple_regression.pdf

30. EQ-5D index calculator. Disponible en: http:// www.economicsnetwork.ac.uk/health/EQ_5D_index_ calculator.xls

31. Saiz Llamosas, José Ramón. Impacto de un programa de fisioterapia sobre la movilidad, el equilibrio y la calidad de vida de las personas mayores. Tesis doctoral. Universidad de Valladolid, 2011.

32. Azpiazu-Garrido M, Cruz-Jentoft A, VillagrassaFerrer JR et al. Calidad de vida en mayores de 65 años no institucionalizados de dos áreas sanitarias de Madrid. Aten Primaria. 2003;31(5):285-94.
33. Gobierno de Navarra: Encuesta de Salud de Navarra del año 2000. Disponible en:

ht t p ://ww w.navarra.es/home_es/Te$\mathrm{mas} / \mathrm{Portal}+\mathrm{de}+\mathrm{la}+\mathrm{Salud} / \mathrm{Profes} \overline{\mathrm{i}}$ onales/ Informacion+tecnica/Sanidad/encuesta + de + salud.htm

34. Bellón JA, Delgado-Sánchez A, Luna J, LardelliClaret P. Patient psychosocial factors and primary care consultations: a cohort study. Fam Pract. 2007;24:5629.

35. Gómez E. Género, equidad y acceso a los servicios de salud: una aproximación empírica. Rev Panam Salud Publica. 2002;11(5-6):327-34

36. Lizán-Tudela L, Reig-Ferrer A. Perspectiva del paciente en la evaluación de resultados en atención primaria: la medida de la calidad de vida relacionada con la salud. Cuadernos de Gestión. 1998;4:119-31.

37. Feeny D, Garner R, Bernier J, Thomson A, McFarland $\mathrm{BH}$, Huguet $\mathrm{N}$ et al. Physical Activity Matters: Associations Among Body Mass Index, Physical Activity and Health-Related Quality of Life Trajectories Over 10 Years. J Phys Act Health. 2013; PMID:24176861 [Epub ahead of print]

38. Weisman CS. Women's health care. Baltimore: The Johns Hopkins University Press; 1998. pp 106110 .

39. Charlson ME, Pompei P, Ales KL, Mackenzie CR. A new method of classifying prognostic comorbidity in longitudinal studies: development and validation. J Chronic Dis. 1987;40:373-83.

40. Mutran E, Ferraro KF. Medical need and use of services among older men and women. J Gerontology: Social Sciences. 1998;5(Suppl 1):62-171.

41. García-Morillo JS, Bernabeu-Wittel M, OlleroBaturone M, Aguilar-Guisad M, Ramírez-Duque N, González de la Puente MA. Incidencia y características clínicas de los pacientes con pluripatología ingresados en una unidad de Medicina Interna. Med Clin (Barc). 2005;125:5-9.

42. Murtagh KN, Hubert HB. Gender differences in physical disability among an elderly cohort. Am J Public Health. 2004;94:1406-11.

43. Saarni SI, Suvisaari J, Sintonen H, Pirkola S, Koskinen S, Aromaa A et al. Impact of psychiatric disorders on health-related quality of life: general population survey. Br J Psychiatry. 2007;190:326-32.

44. Kapur N, Hunt I, Lunt M, McBeth J, Creed F, Macfarlane G. Primary care consultation predictors in men and women: a cohort study. Br J Gen Pract. 2005;55:108-13.

45. Tanner JL, Cockerham WC, Spaeth JL. Predicting physician utilization. Med Care. 1983;21:360-9. 
46. Rodríguez M, Stoyanova A. La influencia del tipo de seguro y la educación en los patrones de utilización de los servicios sanitarios. Gac Sanit. 2004;18(Supl 1):10211.

47. Fernández Merino MC, Vérez VL, Gude SF. Morbilidad crónica y autopercepción de salud en los ancianos de una comunidad rural. Aten Primaria. 1996;17(2):108-12.

48. Oliva-Moreno J, Lopez-Bastida J, Worbes-Cerezo M, Serrano-Aguilar P. Health related quality of life of Canary Island citizens. BMC Public Health. 2010;10:675.

49. Macintyre S, Ellaway A, Cummings S. Place effects on health: how can we conceptualise, operationalise and measure them? Soc Sci Med. 2002;55:125-39.

50. Gattino S, Piccoli N, Fassio O, Rollero Ch. Quality of life and sense of community. A study on health and place of residence. J Community Psychol. 2013;41(7):811-26. 